Giuseppe Rizzo*, Ilenia Mappa, Maria Elena Pietrolucci, Jia Li Angela Lu, Alexander Makatsarya and Francesco D’Antonio

\title{
Effect of SARS-CoV-2 infection on fetal umbilical vein flow and cardiac function: a prospective study
}

https://doi.org/10.1515/jpm-2021-0657

Received December 5, 2021; accepted January 8, 2022;

published online January 21, 2022

\section{Abstract}

Objectives: To explore whether severe acute respiratory syndrome coronavirus 2 (SARS-CoV-2) can affect umbilical vein blood flow (UVBF) and fetal cardiac function.

Methods: Prospective case-control study of consecutive pregnancies complicated by SARS-CoV-2 infection during the second half of pregnancy matched with unaffected women. Measurements of UVBF normalized for fetal abdominal circumference (UVBF/AC), atrial area (AA) and ventricular sphericity indices (SI) were compared between the two study groups. Chi-square and Mann-Whitney U tests were sued to analyze the data.

Results: Fifty-four consecutive pregnancies complicated and 108 not complicated by SARS-CoV-2 infection were included. The median gestational age at infection was 30.2 (interquartile range [IQR] 26.2 34.1). General baseline and pregnancy characteristics were similar between pregnant women with compared to those without SARS-CoV-2 infection. There was no difference in UVBF/AC (study groups z value -0.11 vs. 0.14 control $p$ 0.751) values between pregnancies complicated compared to those not complicated by SARS-CoV-2 infection. Likewise, there was no difference in the left and right AA (left 1.30 vs. $1.28 \mathrm{p}=0.221$ and right 1.33 vs. $1.31 \mathrm{p}=0.324$ ) and SI (left 1.75 vs. $1.77 \mathrm{p}=0.208$ and right 1.51 vs. $1.54 \mathrm{p}=0.121$ ) between the two groups.

*Corresponding author: Giuseppe Rizzo, MD, Department of Obstetrics and Gynaecology, Fondazione Policlinico Tor Vergata, University of Rome Tor Vergata, Rome, Italy, Phone: +39 0620908124 , E-mail: giuseppe.rizzo@uniroma2.it. https://orcid.org/0000-00025525-4353

Ilenia Mappa, Maria Elena Pietrolucci and Jia Li Angela Lu, Department of Obstetrics and Gynecology, Università di Roma Tor Vergata, Rome, Italy

Alexander Makatsarya, The First I.M. Sechenov Moscow State Medical University, Moscow, Russia

Francesco D'Antonio, Department of Obstetrics and Gynecology, Center for Fetal Care and High-Risk Pregnancy, University of Chieti, Chieti, Italy
Conclusions: SARS-CoV-2 infection does not affect UVBF and fetal cardiac function in uncomplicated pregnancies.

Keywords: COVID-19; fetal cardiac function; severe acute respiratory syndrome coronavirus 2 (SARS-CoV-2) infection; umbilical vein flow.

\section{Introduction}

Severe acute respiratory syndrome coronavirus 2 (SARSCoV-2) infection is still the biggest universal health hazard since the starting of pandemic in 2020, with increasing reports of new infection requiring hospitalization, admission to Intensive Care Unit (ICU) and deaths [1].

It has been evidences by systematic reviews and observational studies that pregnancy is an independent risk factor for major complications [2-5]. Indeed pregnant women with SARS-CoV-2 infection show a higher risk of severe respiratory symptoms, need for assisted ventilation and admission to ICU when compared to a non-pregnant population [4, 5]. Furthermore, a large proportion of pregnancies complicated by SARS-CoV-2 infection presents placental histopathological abnormalities including hypoperfusion and inflammation, suggesting a potential connection between infection and complications related to impaired placental function [6-10].

Impaired placental function is associated with a reduction of umbilical vein blood flow (UVBF) since the early stages of pregnancy which leads to changes in fetal cardiac function and morphometry, the so called cardiac remodeling [11-15]. SARS-CoV-2 infection also represents an additional factor for acute cardiovascular disease and altered myocardial function in adults, including acute heart failure, cardiogenic shock, ventricular dysfunction, wall motion abnormalities, global ventricular dysfunction and grade II or III diastolic dysfunction [16,17]. Despite this association, there is no study exploring the effect of SARS-CoV-2 infection on fetal cardiac function.

We hypothesized that SARS-CoV-2 infection may have a direct or non-direct effect on placental and fetal cardiac function.

The primary aim of this study was to compare the UVBF in pregnancies complicated and in those not complicated 
by SARS-CoV-2 infection. The secondary aim was to elucidate whether SARS-CoV-2 infection affects fetal cardiac function.

\section{Materials and methods}

\section{Study population}

This is a secondary analysis of a prospective case-control study SARS-CoV-2 infection in pregnancy [18] including consecutive singleton pregnancies complicated by infection early in gestation receiving antenatal care at the Department of Obstetrics and Gynecology Università di Roma Tor Vergata, Italy from September 2020 to March 2021. Inclusion criteria were 1) gestational age confirmed by crown-rump length at the 11-14 weeks scan, 2) confirmed SARS-CoV-2 infection during pregnancy and 3) delivery in our unit. Pregnancies complicated by fetal structural or chromosomal anomalies, maternal smoking or medical complications potentially affecting fetal hemodynamic (i.e., diabetes, chronic hypertension and autoimmune diseases) were excluded from the analysis. This cohort was compared to a control group of pregnancies unexposed to SARS-CoV-2 followed in our department in the same time interval. The control group had the same exclusion criteria of the study group and was matched with the latter as regard as the main maternal and pregnancy characteristics with a 1:2 ratio. The study was approved by Institutional Review Board of our institution (\#Ost4-2020 30/July 2020) and informed consent was obtained by all the included women.

SARS-CoV-2 infection was confirmed by the presence of positive real-time polymerase-chain reaction (RT-PCR) result obtained by nasopharyngeal swab specimens during pregnancy. All women with confirmed SARS-CoV-2 infection experienced mild symptoms (fever, cough, sore throat, loss of smell and taste, diarrhea) and none required hospitalization.

\section{Ultrasound assessment}

Recordings were performed using a Samsung W80 or Hera W10 ultrasound equipment with a 1-8 MHz volumetric probe. Doppler velocity waveforms and UV diameter were measured at the intraabdominal straight portion of the vessel following a previously reported technique $[11,15]$. Doppler velocity waveforms were recorded for at least $10 \mathrm{~s}$ of uniform flow in periods of fetal quiescence with an angle of insonation as close as possible to $0^{\circ}$ and always below $20^{\circ}$. On the freezed images the time-averaged maximum velocity (TAMXV) was measured.

To measure UV diameter, the vessel was visualized by insonating perpendicularly its intra-abdominal portion. The internal diameter was then measured using the function of the ultrasound equipment designed for the semi-automated assessment of nuchal translucency. As described previously [15], the box for the measurement was placed on all the length of the UV and the inner-inner diameter obtained. UVBF $(\mathrm{mL} / \mathrm{min})$ was calculated as $\pi^{\star}$ (UV diameter $/ 2)^{2} 0.5$ * TAMXV. In order to control the effect of fetal size, UVBF was expressed as a ratio with abdominal circumference (AC) expressed in mm (UVBF/AC). AC was selected as biometric parameter since it better reflects fetal growth and is less prone to inherent errors such as those present in the formulas estimating fetal weight [15].

Fetal echocardiography included a comprehensive anatomical study and a functional evaluation. Based on previous experience we selected among functional indices the areas of the atria and the sphericity index (SI) of both ventricles [11, 17].

Cardiac measurements were obtained from two-dimensional images of the four-chamber view with an angle between $45^{\circ}$ and $90^{\circ}$ between the interventricular septum and to the ultrasound beam following a previously reported technique [12] Images were then optimized to strengthen the borders echogenicty of cardiac walls and then the optimized cine clips were stored. Left and right atrial areas (AA) were taken at maximum point of atrial distension while ventricular base-to-apex lengths and basal diameters at end diastole. Left and right ventricular SI were then calculated as base-to-apex length divided by basal diameter.

\section{Data analysis}

Since Doppler and cardiac indices change with gestational age, data were expressed as the number of standard deviations ( $\mathrm{z}$ value) by which they diverge from the expected mean difference obtained from previously constructed reference limits $[12,15,17,18]$.

A sample size analysis was performed to evaluate the sample size necessary. Given a significance of 0.05 and power of -0.80 a sample size of 50 in the study group and 100 in the control group resulted necessary to demonstrate differences of $0.5 \mathrm{z}$-scores in the variables considered.

The Shapiro Wilk normality test was used to evaluate the distribution of data. Categorical variables were presented as numbers (n) and percentages (\%) and analyzed using Chi-square test, while continuous variables as median and interquartile range (IQR) and analyzed using Mann-Whitney U test.

Data were analyzed using SPSS (version 23.0 IBM Corp. Armonk. NY. USA) and MedCalc Statistical softwares (version 14.8 MedCalc Software bvba, Ostend, Belgium). A two-tailed p-values lower than 0.05 were considered as statistically significant.

\section{Results}

Fifty-four consecutive pregnancies complicated and 108 not complicated by SARS-CoV-2 infection were included in the analysis. Median gestational age at SARS-CoV-2 infection was 30.2 weeks (IQR 28.7-32.4) and all women were asymptomatic with negative RT-PCR swabs at the time of the 36 weeks scan. General baseline and pregnancy characteristics were similar between pregnant women with compared to those without COVID-19 infection (Table 1). Despite a tendency to lower UVBF/AC in women who experienced COVID-19 infection the difference did not achieve statistical significance (study groups $\mathrm{z}$ value -0.11 vs. 0.14 control $\mathrm{p}=0.07$ ) values (Figure 1 ). Likewise, there was no difference in the left and right AA (left 1.30 vs. 1.28 $\mathrm{p}=0.221$ and right 1.33 vs. $1.31 \mathrm{p}=0324$ ) and SI (left 1.75 vs. $1.77 \mathrm{p}=0.208$ and right 1.51 vs. $1.54 \mathrm{p}=0.121$ ) (Table 2). 
Table 1: General characteristics of study population stratified according to the exposure to SARS-CoV-2.

\begin{tabular}{|c|c|c|c|}
\hline Characteristics & $\begin{array}{r}\text { Pregnancies } \\
\text { complicated by } \\
\text { SARS-CoV-2 } \\
\text { infection }(n=54)\end{array}$ & $\begin{array}{r}\text { Pregnancies not } \\
\text { complicated by } \\
\text { SARS-CoV-2 } \\
\text { infection }(n=108)\end{array}$ & $\begin{array}{r}\mathrm{p}- \\
\text { Value }\end{array}$ \\
\hline Maternal age, years & $30.7(29.4-32.3)$ & $30.5(28.1-32.1)$ & 0.624 \\
\hline Maternal height, cm & $162(157-164)$ & $161(158-165)$ & 0.813 \\
\hline $\mathrm{BMI}, \mathrm{kg} / \mathrm{m}^{2}$ & $25.9(24.3-29.1)$ & $26.2(24.1-29.2)$ & 0.345 \\
\hline Ethnicity, n (\%) & & & 0.455 \\
\hline Caucasian & $51(94.4 \%)$ & $103(95.4 \%)$ & \\
\hline Other & $5(5.6 \%)$ & $5(4.6 \%)$ & \\
\hline Nulliparous, n (\%) & $36(66.6 \%)$ & $74(68.5 \%)$ & 0.952 \\
\hline $\begin{array}{l}\text { Assisted conception, } \\
\mathrm{n}(\%)\end{array}$ & $3(6.1 \%)$ & 5 (5.1\%) & 0.797 \\
\hline $\begin{array}{l}\text { Gestational age at US } \\
\text { examination, weeks }\end{array}$ & $36.5(35.3-37.0)$ & $36.6(35.5-36.9)$ & \\
\hline $\begin{array}{l}\text { Gestational age at } \\
\text { COVID-19 infection, } \\
\text { weeks }\end{array}$ & $30.2(26.234 .1)$ & & \\
\hline $\begin{array}{l}\text { Gestational age at } \\
\text { delivery, weeks }\end{array}$ & $39.4(38.0-40.9)$ & $39.9(38.1-40.7)$ & 0.224 \\
\hline Birthweight, g & $\begin{array}{r}3,350 \\
(2,810-3,580)\end{array}$ & $\begin{array}{r}3,410 \\
(2,940-3,790)\end{array}$ & 0.377 \\
\hline Male, n (\%) & 26 (48.1\%) & $55(50.9 \%)$ & 0.816 \\
\hline
\end{tabular}

Data are expressed as median and IQR or $\mathrm{n}$ and \%. IQR, interquartile range; SARS-CoV-2, severe acute respiratory syndrome coronavirus 2 .

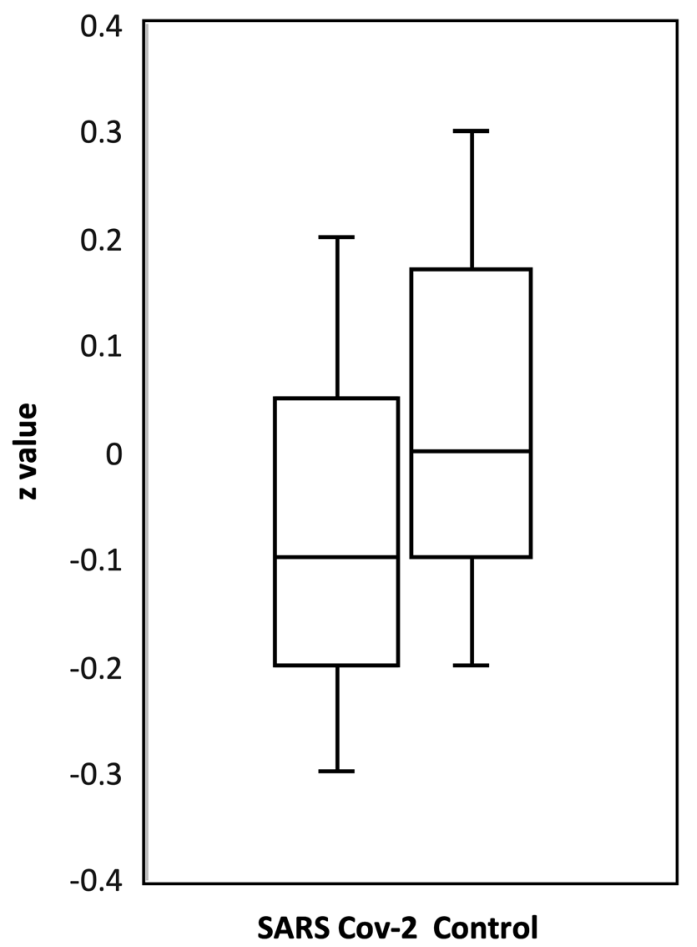

Figure 1: Box-Whisker plots of umbilical vein blood flow/abdominal circumference (UVBF/AC) in fetuses of mothers affected by severe acute respiratory syndrome coronavirus 2 (SARS-CoV-2) and in control fetuses.
Table 2: Comparison of biometric and Doppler indices between the two groups obtained at the time of the two ultrasonographic examination.

\begin{tabular}{lrrr}
\hline Characteristics & $\begin{array}{r}\text { Pregnancies } \\
\text { complicated by } \\
\text { SARS-CoV-2 }\end{array}$ & $\begin{array}{r}\text { Pregnancies not } \\
\text { complicated by } \\
\text { SARS-CoV-2 }\end{array}$ & $\begin{array}{r}\mathbf{p}- \\
\text { Value } \\
\text { infection }(\mathbf{n}=54)\end{array}$ \\
\hline infection $(\mathbf{n}=108)$ & \\
\hline RVBF/AC z value & $-0.07(-0.63-0.56)$ & $0.18(-0.34-0.76)$ & 0.229 \\
LA area z value & $-0.04(-0.54-0.9)$ & $0.01(-0.48-0.89)$ & 0.526 \\
LV sphericity & $0.36(-0.15-095)$ & $0.23(-0.48-0.70)$ & 0.163 \\
index & $0.19(-0.65-0.68)$ & $0.25(-0.24-0.69)$ & 0.168 \\
RV sphericity & $0.06(-0.40-0.60)$ & $0.14(-0.37-0.62)$ & 0.183 \\
index & & & \\
\hline
\end{tabular}

Data are expressed as z values. Data are expressed as median and IQR. IQR, interquartile range; UVBF/AC, umbilical vein blood flow/ abdominal circumference; RA, right atrium; LA, left atrium; LV, left ventricle; $R V$, right ventricle; SARS-CoV-2, severe acute respiratory syndrome coronavirus 2 .

\section{Discussion}

\section{Main findings}

The findings from this study showed that SARS-CoV-2 infection does not significantly affect UBVF. Despite a tendency towards lower values of UVBF values in affected pregnancies the difference does not reach the statistical significance when compared to pregnancies not exposed to the virus. Likewise, there was no differences in fetal cardiac function, suggesting that SARS-CoV-2 infection in pregnancy is unlikely to affect fetal venous and cardiac hemodynamic, at least in uncomplicated pregnancies.

\section{Strength and limitations}

To the best of our knowledge, this is the first study evaluating the effects of SARS-CoV-2 infection on fetal venous and cardiac function. The main strength of our study was that data were collected prospectively on relatively large population following a comprehensive study protocol that included the assessment of umbilical vein circulation and fetal cardiac hemodynamic. Furthermore, the two study populations were controlled as regard the main maternal and pregnancy variables potentially affecting fetal hemodyamics.

The main limitation of this study relies in the inclusion of only mildly symptomatic cases, raising the possibility of a different behavior on presence of more severe diseases. Indeed, severe SARS-CoV-2 infection has been associated 
with higher risk of vasculopathy and we cannot exclude that the lack of association between SARS-CoV-2 infection and the variables tested may be due to the fact that we included only pregnant women in the mild spectrum of the disease. However, about $92-95 \%$ of pregnant women with SARS-CoV-2 infection does not experience the severe spectrum of the disease allowing to apply the findings for our study to the large majority of pregnant women acquiring the infection in pregnancy [19]. Furthermore, we restricted our observation to infection occurring during at mid gestation, and we cannot exclude that the findings could be different, if the infection had occurred earlier in pregnancy. Finally, we included only pregnancies not complicated by placental insufficiency. Pregnancies complicated by impaired placental function and fetal growth restriction show an altered cardiac geometry and hemodynamic induced by hypoxemia. In this scenario, the magnitude of hemodynamic and cardiac changes induced by the virus may be potentially more significant in fetuses already showing cardiac dysfunction due to placental insufficiency.

\section{Comparison with other studies}

The well documented association between SARS-CoV-2 infection and placental lesions due to inflammation and hypoperfusion [10] questions whether fetuses from infected mothers are at risk of developing similar change in venous hemodynamic and cardiac morphometry reported in fetal growth restriction due to placental insufficiency. There is now a wealth of consistent published data showing a higher prevalence of signs of decidual arteriopathy in pregnant women with SARS-CoV-2 infection, suggesting a direct link between infection and impaired placental function $[9,10]$. The potential mechanisms responsible for the higher risk of fetal compromise or death in pregnancy may be primarily explained on the basis of a secondary effect of the virus due to placenta hypoperfusion induced by the compromised hemodynamic status of the mother, or alternatively, of an increase pro-inflammatory mediators induced by the virus at placental level [20].

However, there is another potential mechanism of cardiovascular dysfunction related to SARS-CoV-2 infection. In adults, SARS-CoV-2 infection has been reported to induce direct or indirect cardiac damage resulting in right ventricular dysfunction, left ventricular wall motion abnormalities, global left ventricular dysfunction and grade II or III diastolic dysfunction [21]. These changes represent the pathophysiological bases of the higher occurrence of acute heart failure, cardiogenic shock, myocardial ischemia or infarction, stress cardiomyopathy and arrhythmias in these patients. There are also recent reports on the presence of myocarditis in newborns from SARS-CoV-2 infection suggesting a direct effect of the virus on fetal heart before birth $[22,23]$.

The Host Cell Receptor for SARS-CoV-2 is the biologically critical enzyme ACE2 receptor, which is involved in ventricular remodeling in many pathological conditions, thus making the infection a potential trigger for cardiovascular dysfunction. These receptors are also expressed on fetal cardiomyocyte, thus making the fetal heart a potential target for infection.

The lack of associations between SARS-CoV-2 and altered cardiac function observed in our series may be explained on different basis. First, the burden of cardiovascular changes due to SARS-CoV-2 observed in adults and children has been related to the severity of the infection. In the present series, we included only asymptomatic or mildly asymptomatic cases, thus potentially overlooking the actual role of the virus in severe infection. Second, cardiovascular changes related to placental insufficiency are induced by prolonged chronic hypoxemia and commonly presents in the third trimester of pregnancy. In our series, we included only uncomplicated pregnancies and it may be plausible that the effect of the virus on fetal cardiac function may be more pronounced in pregnancies already complicated by classic placental insufficiency and fetal growth restriction. Last, the lack of cardiovascular and hemodynamic changes related to the infection may be in view of the relative immaturity and lower concentration of ACE2 receptor in the human fetus.

\section{Implications for clinical practice}

The Royal College of Obstetricians and Gynaecologists recently suggested that pregnant women recovering from SARS-CoV-2 infection should be offered only in presence of a severe disease a fetal growth scan approximately 14 days after recovery from their illness, while in asymptomatic or mild form cases no changes in routine antenatal care is required [24].

The findings from our studies support this recommendation showing that pregnancies complicated by mild SARS-CoV-2 infection are not at higher risk of hemodynamic and cardiac changes, and therefore they do not require additional scans through pregnancy to rule out these disorders, unless pre-existing complications are present.

Therefore, women with mild SARS-CoV-2 infection should be counseled and reassured about their low risk of 
adverse fetal outcome [25]. This is particularly important since pregnant women with SARS-CoV-2 infection experience increased anxiety levels due to their specific concerns about the detrimental effect of the infection on their fetus $[26,27]$.

\section{Conclusions}

There are no evidences that SARS-CoV-2 infection induce cardiovascular dysfunction in uncomplicated pregnancies. The findings from this study do not support a policy of increased fetal surveillance and elective induction of labor due to the infection. Further studies are needed in to elucidate the influence and the magnitude of SARS-CoV-2 infection on fetal cardiac function and hemodynamic in pregnancies complicated by the severe spectrum of the diseased and in women already affected by impaired placental function and/or fetal growth restriction.

Research funding: None declared.

Author contributions: All authors have accepted responsibility for the entire content of this manuscript and approved its submission.

Competing interests: Authors state no conflict of interest. Informed consent: Informed consent was obtained from all individuals included in this study.

Ethical approval: The study was approved by Institutional Review Board of our institution (\#Ost4-2020 30/July 2020).

Data availability: The datasets analyzed during the current study are available from the corresponding author on reasonable request.

\section{References}

1. Centers for Disease Control and Prevention (CDC). Data on COVID-19 during pregnancy: weekly COVID-19 pregnancy data. Available from: https://www.cdc.gov/coronavirus/2019-ncov/ cases-updates/special-populations/pregnancy-data-on-covid19.html [Accessed 4 Dec 2021].

2. Di Mascio D, Khalil A, Saccone G, Rizzo G, Buca D, Liberati M, et al. Outcome of coronavirus spectrum infections (SARS, MERS, COVID-19) during pregnancy: a systematic review and meta-analysis. Am J Obstet Gynecol MFM 2020;2: 100107.

3. Khalil A, Kalafat E, Benlioglu C, O'Brien P, Morris E, Draycott T, et al. SARS-CoV-2 infection in pregnancy: a systematic review and meta-analysis of clinical features and pregnancy outcomes. EClinicalMedicine 2020;25:100446.

4. Di Mascio D, WAPM (The World Association of Perinatal Medicine) Working Group on COVID-19. Maternal and perinatal outcomes of pregnant women with SARS-COV-2 infection. Ultrasound Obstet Gynecol 2021;57:232-41.

5. Di Mascio D, Sen C, Saccone G, Galindo A, Grünebaum A, Yoshimatsu J, et al. Risk factors associated with adverse fetal outcomes in pregnancies affected by Coronavirus disease 2019 (COVID-19): a secondary analysis of the WAPM study on COVID-19. J Perinat Med 2020;49:111-5.

6. Malinowski AK, Noureldin A, Othman M. COVID-19 susceptibility in pregnancy: immune/inflammatory considerations, the role of placental ACE-2 and research considerations. Reprod Biol 2020; 20:568-72.

7. Dhaundiyal A, Kumari P, Jawalekar SS, Chauhan G, Kalra S, Navik U. Is highly expressed ACE 2 in pregnant women "a curse" in times of COVID-19 pandemic? Life Sci 2021;264:118676.

8. Shanes ED, Mithal LB, Otero S, Azad HA, Miller ES, Goldstein JA. Placental pathology in COVID-19. Am J Clin Pathol 2020;154: 23-32.

9. Schwartz DA, Baldewijns M, Benachi A, Bugatti M, Collins RRJ, De Luca $D$, et al. Chronic histiocytic intervillositis with trophoblast necrosis are risk factors associated with placental infection from coronavirus disease 19 (COVID-19) and intrauterine maternal-fetal systemic acute respiratory coronavirus 2

(SARSCoV-2) transmission in liveborn and stillborn infants. Arch Pathol Lab Med 2021;45:517-28.

10. Di Girolamo R, Khalil A, Alameddine S, D’Angelo E, Galliani C, Matarrelli B, et al. Placental histopathology after SARS-CoV-2 infection in pregnancy: a systematic review and meta-analysis. Am J Obstet Gynecol MFM 2021;3:100468.

11. Rizzo G, Mattioli C, Mappa I, Bitsadze V, Khizroeva J, Stodki M, et al. Hemodynamic factors associated with fetal cardiac remodeling in late fetal growth restriction: a prospective study. J Perinat Med 2019;47:683-8.

12. Rizzo G, Pietrolucci ME, Mappa I, Bitsadze V, Khizroeva J, Makatsariya A, et al. Fetal cardiac remodeling is affected by the type of embryo transfer in pregnancies conceived by in vitro fertilization: a prospective cohort study. Fetal Diagn Ther 2020;13:1-7.

13. Rizzo G, Capponi A, Pietrolucci ME, Capece A, Arduini D. Firsttrimester umbilical vein blood flow in pregnancies with low serum pregnancy-associated plasma protein-A levels: an early predictor of fetal growth restriction. Ultrasound Obstet Gynecol 2010;36: 433-8.

14. Rizzo G, Mappa I, Bitsadze V, Stodki M, Khizroeva J, Makatsariya $A$, et al. Role of first-trimester umbilical vein blood flow in predicting large-for-gestational age at birth. Ultrasound Obstet Gynecol 2020;56:67-72.

15. Rizzo G, Mappa I, Bitsadze V, Stodki M, Khizroeva J, Makatsariya A, et al. Role of Doppler ultrasound at time of diagnosis of late-onset fetal growth restriction in predicting adverse perinatal outcome: prospective cohort study. Ultrasound Obstet Gynecol 2020;55: 793-8.

16. Rizzo G, Mappa I, Maqina P, Bitsadze V, Khizroeva J, Makatsarya A, et al. Effect of SARS-CoV-2 infection during the second half of pregnancy on fetal growth and hemodynamics: a prospective study. Acta Obstet Gynecol Scand 2021;100:1034-9.

17. García-Otero L, Soveral I, Sepúlveda-Martínez Á, Rodriguez-López M, Torres X, Guirado L, et al. Reference ranges for fetal cardiac, ventricular and atrial relative size, sphericity, ventricular dominance, wall asymmetry and relative wall thickness from 18 to 41 gestational weeks. Ultrasound Obstet Gynecol 2021; 58:388-97. 
18. Rizzo G, Rizzo L, Aiello E, Allegra E, Arduini D. Modelling umbilical vein blood flow normograms at 14-40 weeks of gestation by quantile regression analysis. J Matern Fetal Neonatal Med 2016; 29:701-6.

19. Yanes-Lane M, Winters N, Fregonese F, Bastos M, Perlman-Arrow S, Campbell JR, et al. Proportion of asymptomatic infection among COVID-19 positive persons and their transmission potential: a systematic review and meta-analysis. PLoS One 2020;15: e0241536.

20. Kotlyar AM, Grechukhina O, Chen A, Popkhadze S, Grimshaw A, Tal 0, et al. Vertical transmission of coronavirus disease 2019: a systematic review and meta-analysis. Am J Obstet Gynecol 2021; 224:35-53.e3.

21. Rind IA, Cannata A, McDonaugh B, Cassimon B, Bannister C, Scott $P A$, et al. Patients hospitalised with heart failure across different waves of the COVID-19 pandemic show consistent clinical characteristics and outcomes. Int J Cardiol 2021;29:S0167-5273.

22. Miraskari R, Sayarifard E, Kharrazi H, Naserfar N, Sayarifard A. Neonatal SARS-CoV-2 infection and congenital myocarditis: a case report and literature review. Arch Pediatr Infect Dis 2020;8: e103504.

23. Azeka E, Arshad A, Martins C, Dominguez AC, Siqueira A, Loss AS, et al. Case report: dilated cardiomyopathy in a newborn, a potential association with SARS-COV-2. Front Pediatr 2021;9: 674300.

24. The Royal College of Obstetricians and Gynaecologists, The Royal College of Midwives. Coronavirus (COVID-19) infection in pregnancy. Available from: https://www.rcog.org.uk/ coronavirus-pregnancy [Accessed 4 Dec 2021].

25. Di Mascio D, Buca D, Berghella V, Khalil A, Rizzo G, Odibo A, et al. Counseling in maternal-fetal medicine: SARS-CoV-2 infection in pregnancy. Ultrasound Obstet Gynecol 2021;57:687-97.

26. Mappa I, Distefano FA, Rizzo G. Effects of coronavirus 19 pandemic on maternal anxiety during pregnancy: a prospectic observational study. J Perinat Med 2020;48:545-50.

27. Thapa SB, Mainali A, Schwank SE, Acharya G. Maternal mental health in the time of the COVID-19 pandemic. Acta Obstet Gynecol Scand 2020;99:817-8. 